\title{
Verschlüsselung in Wittgensteins Nachlass
}

\author{
Ilse Somavilla, Innsbruck
}

Im Jahre 1929 notierte Wittgenstein im MS 106, 4:

Es ist merkwürdig welche Erleichterung es mir ist manches in einer geheimen

Schrift nieder zu schreiben was ich nicht gerne lesbar schreiben möchte. ${ }^{1}$

Ausgehend von dieser Notiz und weiteren Bemerkungen und im Hinblick auf Wittgensteins Schweigen über bestimmte Bereiche in der Philosophie, möchte ich in diesem Beitrag der Frage nachgehen, ob Wittgenstein in der Verschlüsselung von Texten eine Möglichkeit sah, über Dinge zu sprechen bzw. zu schreiben, über die er eigentlich schweigen wollte. Dies betrifft sowohl philosophische als auch persönliche, existentielle Fragen, deren Verbalisierung, so scheint es, er dem oberflächlichen „Schnellleser“ vorenthalten wollte. Für diese Annahme spricht eine Eintragung vom 9.2.1937 im MS 157a:

Es ist ein großer Unterschied zwischen den Wirkungen einer Schrift die man leicht \& fließend lesen kann \& einer die man schreiben aber nicht leicht entziffern/lesen kann. Man schließt, in ihr die Gedanken ein, wie in einer Schattulle.

$\mathrm{Ob}$, und wenn ja, welche Gedanken Wittgenstein mittels Verschlüsselung bewahren, schützen, ja verschließen wollte, soll sich aus den folgenden Untersuchungen zeigen.

Chronologisch betrachtet, sind uns erste Aufzeichnungen in Code aus der Zeit des Ersten Weltkriegs bekannt, doch wie auch McGuinness mutmaßt, spricht die relativ einfache Handhabung des Code $^{2}$ dafür, dass Wittgenstein schon früher von diesem Gebrauch machte. Wie ein Brief seiner Schwester Helene zeigt, dürfte der Code innerhalb der Familie Witt-

${ }^{1}$ Im Folgenden sind codierte Stellen kursiv gesetzt.

${ }^{2}$ Dieser besteht mehr oder weniger in der Umkehrung des Alphabets: $a=z, b=y$ etc. (Als kleine Abweichung sei der Buchstabe r erwähnt, der sowohl i als auch j bedeutet). 
genstein, vermutlich unter den Geschwistern, als eine Art Spielerei mit Schrift verwendet worden sein.

Abgesehen von den Kriegstagebüchern 1914-1916, die bekannterweise in codierte und nicht codierte Aufzeichnungen - auf den jeweils linken und rechten Seiten - unterteilt sind, kommen codierte Bemerkungen auch in einem Tagebuch der Dreißigerjahre sowie verstreut über den ganzen Nachlass vor: hier als vereinzelte, häufig in Form von Fragmenten oder Aphorismen auftretende Bemerkungen, die mit dem philosophischen Kontext der Manuskripte augenscheinlich nichts $\mathrm{zu}$ tun haben, sondern vielmehr aus diesem als etwas Eigenständiges herausragen. Insgesamt gibt es im Nachlass an die 450 Stellen codierte Bemerkungen, wobei es sich manchmal nur um einzelne Sätze oder kurze Absätze, zuweilen aber auch um längere, sich über mehrere Seiten erstreckende Passagen handelt.

Mehr oder weniger grob gesprochen, kann der Großteil dieser Bemerkungen persönlichen, tagebuchartigen Aufzeichnungen sowie der Thematik des „unaussprechlichen Bereichs“ - also Ethik, Ästhetik und Religion - zugeordnet werden. Häufig finden sich darunter Reflexionen kulturgeschichtlichen Inhalts, aber auch philosophische Gedankengänge. Oft berichtet Wittgenstein in Code über die Art und Weise seines Philosophierens - gleich einer kritischen Stimme von außerhalb bzw. aus der Perspektive einer Meta-Ebene. Interessanterweise verfasste er die Instruktionen für die Veröffentlichung seiner Schriften in Code, was dafür spricht, dass er sich der Einfachheit der Entzifferung seines Code offenbar bewusst war. Insofern sollte dieser nicht als Geheimschrift bezeichnet werden.

Obwohl eine eindeutige Zuordnung zu bestimmten Themen problematisch erscheint, wage ich aus bisherigen Untersuchungen zu behaupten, dass Wittgensteins Aufzeichnungen in Code nicht zufällig erfolgten, sondern in seinem Oeuvre als eigener Texttypus zu betrachten sind, dem eine besondere Stellung - auch und gerade in seinem Philosophieverständnis zukommt. Man könnte in diesem Zusammenhang auch auf die Bedeutung von Symbolen und Zeichen sowie von Mimik und Gestik verweisen, die in Wittgensteins Philosophie eine wichtige Rolle spielen und im Grunde eine weitere Form von Verschlüsselung der Ausdrucksformen in der zwischenmenschlichen Kommunikation darstellen.

Im Hinblick auf die Frage, ob formal und semantisch interne Analogien unter diesen Stellen bestehen und ob eine Zuordnung zu bestimmten 
Aspekten unter einer gemeinsamen kulturgeschichtlichen Perspektive zulässig und sinnvoll ist - ob demnach die Annahme einer eigenen Textsorte in Wittgensteins Werk gerechtfertigt ist -, sei auf folgende Kriterien hingewiesen:

A) Inhalt: Hier sind persönliche, tagebuchartige Aufzeichnungen, Bemerkungen ethischen und religiösen Inhalts sowie kulturgeschichtliche und politische Reflexionen anzuführen.

B) Stil: In stilistischer Hinsicht lassen sich folgende Merkmale feststellen, die den Unterschied zu den Aufzeichnungen in Normalschrift aufweisen:

a) ein erzählender bzw. narrativer Ton, der die autobiographischen Aufzeichnungen kennzeichnet.

b) ein lyrischer Stil, der sich bei spontan auftretenden, poetisch anmutenden Bemerkungen inmitten des in nüchternem Ton geführten philosophischen Diskurses zeigt.

c) ein sehr persönlicher, leidenschaftlicher Ton, der sich insbesondere in den Eintragungen religiösen und ethischen Inhalts beobachten lässt.

d) ein Stil in der Art von Gebeten, häufig in der „Möge“- Form: „Möge Gott mir helfen“ etc.

e) ein monologischer Charakter: nicht nur die autobiographischen Notizen, sondern auch solche über ethisch- religiöse und kulturelle Themen in codierter Schrift sind von monologischem Charakter bestimmt und heben sich - gleich Selbstgesprächen - vom dialogischen, häufig an ein fiktives $\mathrm{Du}$ gerichteten, in normaler Schrift gehaltenen Diskurs, ab.

C) Code als „kritische Stimme“ aus der Perspektive einer Meta-Ebene, die in persönlicher Hinsicht sein moralisches Verhalten, in philosophischer Hinsicht, wie erwähnt, die Art und Weise seines Philosophierens - Erfolge und Scheitern - kommentiert und beurteilt.

D) Zeit und Ort: Zahlreiche codierte Bemerkungen sind äußeren Umständen wie der Zeit des Ersten Weltkriegs oder den Aufenthalten in der Einsamkeit in Norwegen usw. zuzuordnen. 
All diese Aspekte sollen im Folgenden gemäß Wittgensteins persönlichen, tagebuchartigen Aufzeichnungen (in den Tagebüchern 1914-1916 und den Tagebüchern 1930-1932/1936-1937) sowie den über den ganzen Nachlass verstreuten Bemerkungen ethischer, ästhetischer und religiöser Thematik erörtert werden.

\section{Tagebücher}

Laut einer Notiz im Jahre 1929 begann Wittgenstein während seiner Zeit in Berlin, Tagebuch-Eintragungen zu machen: „Es war ein für mich wichtiger Schritt", bemerkte er. Unter den Gründen dafür nannte er ,zum Teil““ den „Nachahmungstrieb“ (Gottfried Keller), „zum Teil, das Bedürfnis, etwas von [sich] niederzulegen (also Eitelkeit)“ und ,zum Teil“ als „Ersatz für einen Menschen, dem [er sich] anvertrauen könnte“ (MS 107, 74). Im weiteren Verlauf seiner Gedanken über das Tagebuchschreiben bemerkte er: „Was sich nicht schreiben lässt, lässt sich nicht schreiben“ - er war sich also auch hier wie in der Philosophie der Grenzen von Sprache bewusst. Im selben Jahr begann er, sich mit dem Gedanken einer Autobiographie auseinanderzusetzen, um, wie er bemerkte, sein Leben einmal klar ausbreiten zu können, um es klar vor sich zu haben und auch für andere. „Nicht so sehr um darüber Gericht zu halten als um jedenfalls Klarheit und Wahrheit zu schaffen" (MS 108, 46).

Die Suche nach Klarheit und Wahrheit lässt sich also nicht nur in seinen philosophischen, sondern auch in seinen verschlüsselten Aufzeichnungen verfolgen - hier als eine Auseinandersetzung mit persönlichen Problemen, zumeist moralischer Art. Doch auch die Auseinandersetzung mit ästhetischen Fragen, meist innerhalb seiner Reflexionen über Werte der Literatur und Kunst, über die Wahrhaftigkeit ihres Inhalts, ist an einem hohen Ethos orientiert. Insbesondere Lyrik und Musik, als Medien, das Unaussprechliche auszudrücken, wurden von Wittgenstein hochgeschätzt. So bemerkte er hinsichtlich eines Gedichtes von Ludwig Uhland in einem Brief an Paul Engelmann:

Das Uhlandsche Gedicht ist wirklich großartig. Und es ist so: Wenn man sich nicht bemüht das Unaussprechliche auszusprechen, so geht nichts verloren. Sondern das Unaussprechliche ist, - unaussprechlich - in dem Ausgesprochenen enthalten! (Engelmann, 24). 
In seinem Tagebuch der Dreißigerjahre kam er mehrmals auf die Musik zu sprechen, die in seinem Leben - wie in seiner Philosophie - eine zentrale Stelle einnahm. So hegte er u.a. den Wunsch, durch die Musik sein Leben ausdrücken zu können (DB, 9f.), und zu M. O' C. Drury soll er bemerkt haben, dass es ihm unmöglich sei, in seinem Buch (den Philosophischen Untersuchungen) nur ein einziges Wort zu sagen über alles das, was die Musik für ihn in seinem Leben bedeutet habe (Rhees 1992, 120) - ein Versagen, das er mit dem Unverstandensein seines Werkes in Verbindung brachte.

Aus zahlreichen Bemerkungen Wittgensteins wird der Zusammenhang von Leben, Sprache, Kunst, Philosophie und moralischen Werten deutlich. Wie er selbst festhielt:

Die Denkbewegung in meinem Philosophieren müßte sich in der Geschichte meines Geistes, seiner Moralbegriffe \& dem Verständnis meiner Lage wiederfinden lassen (DB, 125r).

\subsection{Tagebücher 1914-1916}

Als geschlossenste Form von bisher bekannten Aufzeichnungen-in-Code sind die persönlichen Eintragungen der Kriegstagebücher 1914 bis 1916 zu nennen. Es handelt sich dabei um drei Manuskriptbände - MS 101, 102 und 103, gemäß der Katalogisierung von G.H. von Wright. ${ }^{3}$ Die erhaltenen Manuskriptbände bestehen aus Wittgensteins philosophischen Aufzeichnungen in Normalschrift (auf der jeweils rechten Seite, beginnend mit 22.8.1914 und endend mit 10.1.1917) und aus seinen persönlichen Aufzeichnungen in Code, auf der jeweils linken Seite. Während der in Normalschrift gehaltene philosophische Teil der Tagebücher bereits 1960 veröffentlicht wurde ${ }^{4}$, ließen die Herausgeber den in verschlüsselter Schrift geschriebenen, persönlichen Teil weg. Auch in einer für Universitäten käuflichen Mikroverfilmung des Nachlasses wurden die linken Seiten abgedeckt.

${ }^{3}$ Von Wright und McGuinness vermuten allerdings, dass es noch drei weitere Notizbücher aus der Zeit des Ersten Weltkriegs gab, die verloren gegangen sind (McGuinness 1992, 331).

${ }^{4}$ In: Schriften I, Frankfurt: Suhrkamp, 1960. Die verbesserte und um einige Bemerkungen erweiterte Neuausgabe erschien 1979 bei Basil Blackwell, Oxford. 
1982 veröffentlichte Wilhelm Baum Teile aus den verschlüsselten Kriegstagebüchern (auf die er in den sogenannten „Gmundner Notizbüchern" im Tübinger Wittgenstein-Archiv gestoßen war) in der Zeitschrift für katholische Theologie. 1985 erfolgte eine deutsch-spanischkatalanische Edition des gesamten Textes in der philosophischen Zeitschrift Saber, 1990 die deutsche Veröffentlichung bei Turia \& Kant.

Die illegale Edition der Geheimen Tagebücher erregte in der Wittgenstein-Rezeption großes Aufsehen; das bisher bestehende „Bild“ von Wittgenstein als das eines kühl und sachlich argumentierenden Philosophen war erschüttert worden. Andererseits führte der Einblick in Wittgensteins persönliche Probleme zu einem beginnenden Verständnis für die Verknüpfung von Leben und Werk. Allerdings kam es auch zu einer vermehrten Bildung von Mythen um Wittgenstein, bis $\mathrm{zu}$ einer verzerrten Darstellung seiner Persönlichkeit.

Die Trennung zwischen Persönlichem und Philosophischem durch unterschiedliche Schriftarten wird in den Kriegstagebüchern besonders deutlich. Trotzdem gilt dies nicht für alle Aufzeichnungen, sondern es gibt Ausnahmen: d.h. Wittgenstein führt manchmal persönliche Bemerkungen in Normalschrift, wie andererseits philosophische Eintragungen in verschlüsselter Schrift. So beginnt er im ersten Manuskriptband am 9.8.1914 mit persönlichen Aufzeichnungen in Normalschrift, die er ab dem 15.8., mitten in einem in Normalschrift begonnenen Satz, in Code fortsetzt. Seine philosophischen Gedankengänge trägt er erst ab dem 22.8.1914 ein. Laut McGuinness begann er mit dem Code, als er in feindliches Gebiet vordrang. ${ }^{5}$ Dies mag vielleicht für seine ganz persönlichen, vorwiegend moralischen Probleme gelten, doch meines Erachtens hat das Schreiben in verschlüsselter Schrift vor allem mit der Thematik des unaussprechlichen Bereichs zu tun, dem Wittgenstein sich während des Krieges mehr und mehr zu nähern begann. In dieser Zeit, als er sich mit moralischen und religiösen Problemen auseinandersetzte, trug er seine Gedanken darüber in verschlüsselter Schrift auf der jeweils linken Seite ein, wo er Persönliches festhielt in Gegenüberstellung zu den philosophischen Aufzeichnungen in Normalschrift auf den rechten Manuskriptseiten. Trotzdem zeigt sich vom Inhalt her an manchen Stellen allmählich ein Ineinanderüberfließen. Das heißt,

${ }^{5}$ Vgl. McGuinness 1992, 332. 
philosophisches Gedankengut ist auch im persönlichen Teil enthalten, wobei es oft um Wesentliches geht, das in den philosophischen Tagebüchern näher ausgeführt und im Tractatus dann zu Kernaussagen komprimiert wird: So trägt Wittgenstein den Satz „Was sich nicht sagen lässt, lässt sich nicht sagen!" erstmals in Geheimschrift ein. ${ }^{6}$ Am selben Tag ist in den philosophischen Tagebüchern zu lesen: „Ist nicht dies der Grund, warum Menschen, denen der Sinn des Lebens nach langen Zweifeln klar wurde, warum diese dann nicht sagen konnten, worin dieser Sinn bestand“" (vgl. auch TLP, 6.521).

Am selben Tag also, als er sich Gedanken über den Sinn des Lebens machte, den er außerhalb der Welt der Tatsachen - im Bereich des Unaussprechlichen - gelegen sah, trug er sozusagen als Fazit über die Einsicht der Unmöglichkeit, diese Problematik zur Sprache zu bringen, diesen Satz ein. Am 8.7. schreibt er im philosophischen Teil: „An einen Gott glauben heißt, die Frage nach dem Sinn des Lebens verstehen. An einen Gott glauben heißt sehen, daß es mit den Tatsachen der Welt noch nicht abgetan ist $[\ldots]^{] "}$.

Es folgen weitere Reflexionen über den Sinn des Lebens, über Gott, den Willen, das Gewissen, das Schicksal, den Tod, über Zeit und Gegenwart. In diesen Aufzeichnungen wird Wittgensteins Annäherung an einen persönlichen Gott - wie er ihn im codierten Teil, durch die im Krieg erfahrene Nähe des Todes, und verstärkt durch den Einfluss der Lektüre von Tolstois Kurzer Darlegung des Evangelium, bereits des öfteren anspricht deutlich, doch sind auch noch panentheistische, offenbar auf Schopenhauer und Spinoza zurückzuführende Tendenzen, spürbar. Während diese vor allem den früheren Teil der philosophischen Tagebücher prägten, zeichnet sich nun mehr und mehr eine religiöse Haltung ab, die einem personalen Gott gilt. Weitere mögliche Einflüsse von Tolstoi, die anfangs Wittgensteins persönliches Empfinden, also sein Leben in existentieller Hinsicht

6 7.7.1916. In den philosophischen Tagebüchern wird dieser Gedanke erst am 15.10.1916 und in etwas abgeänderter Form festgehalten: „Was man sich nicht denken kann, darüber kann man auch nicht reden. Im Tractatus, 5.61 heißt es dann: „Was wir nicht denken können, das können wir nicht denken; wir können also auch nicht sagen, was wir nicht denken können." - Allerdings bemerkt Wittgenstein am 22.11.1914, dass er an einer Stelle wieder etwas auszudrücken versuche, ,was sich nicht ausdrücken läßt“، 
berührten, doch auch in seine philosophischen Gedankengänge aufgenommen werden, sind die des Lebens in der Gegenwart, nicht in der Zeit (8.7.16), sowie seine Reflexionen über die Rechtfertigung eines glücklichen Lebens „der Not der Welt zum Trotz“, das er des weiteren im Guten und im Schönen gewährleistet sieht.

„Nur wer nicht in der Zeit, sondern in der Gegenwart lebt, ist glücklich. Für das Leben in der Gegenwart gibt es keinen Tod“, notiert er am 8.7.1916 in sein philosophisches Tagebuch. Dieses ewige Leben, das durch ein ganz der Gegenwart gewidmetes Leben erreicht wird, und worunter Unzeitlichkeit, nicht unendliche Zeitdauer zu verstehen ist, ist das gute und ethische Leben. Bei Einsicht der verschlüsselten Tagebücher finden sich dem Inhalt nach dieselben Gedanken, die aus der Erfahrung des Krieges, in höchster existentieller Not durch die ständige Nähe des Todes, entstanden sind. So heißt es am 4.5.1916, also ca. zwei Monate vor der Auseinandersetzung mit dem Leben in der Gegenwart, im codierten Teil: „[...] Dann wird für mich erst der krieg anfangen. Und kann sein - auch das leben! Vielleicht bringt mir die Nähe des Todes das licht des lebens! [...]“ Und ein paar Tage später bemerkt er, dass der Tod dem Leben erst seine Bedeutung gebe (9.5.16).

Im philosophischen Teil resümiert er über den Zweck des Daseins, den er im Anklang an Dostojewski im Leben selbst erfüllt sieht: als ein Leben in der Gegenwart, damit auch im Ewigen. Am 20.7.1916 wechselt er inmitten von analytischer Betrachtungsweise hinsichtlich komplizierter Satz-Operationen zu folgender Äußerung: „Der Mensch kann sich nicht ohne weiteres glücklich machen. Wer in der Gegenwart lebt, lebt ohne Furcht und Hoffnung“" (20.7.16).

Die Auffassung vom Leben in der Gegenwart ohne Furcht vor dem Tode (die er als „Zeichen eines falschen, d.h. schlechten Lebens“ sieht) entspricht auch seiner Bemerkung „Die Welt des Glücklichen ist eine andere als die des Unglücklichen“ (TLP, 6.43), worüber er im Zuge seiner Auseinandersetzung über ,gut" und „,böse“ reflektiert.

Die gelassene Haltung gegenüber den „Äußerlichkeiten“ des Lebens hofft Wittgenstein durch eine verstärkte Hinwendung zum Geistigen zu erreichen, wie auch durch das spinozistisch anmutende Sich-ergeben in Gott und das Schicksal. Alle diese Aspekte bestimmen sowohl die in verschlüsselter Schrift wie die in Normalschrift verfassten philosophischen 
Eintragungen, die bekannterweise die Vorarbeit zur Logisch-philosophischen Abhandlung bildeten.

$\mathrm{Zu}$ beachten ist, dass diese Gedanken zuerst im verschlüsselten Teil vorkommen und erst später - 1916, im 3. Manuskript - im philosophischen Teil. So gesehen wird deutlich, welche Gedankengänge Wittgenstein - in Ausnahmezuständen wie dem des Krieges - bewegten und wie diese schließlich auch seine philosophische Arbeit beeinflussten. Denn während es ihm früher in seiner Auseinandersetzung mit Sprache um die logische Darstellung ging, er sich mit sinnvollen und nicht sinnvollen Sätzen auf analytischer Ebene auseinandersetzte, so kreisen seine Reflexionen mit der Zeit zunehmend auf einer Ebene, die über die Welt der Tatsachen hinausreicht, wo es um das Problematische des Sinns des Daseins geht, der jedoch - als zu metaphysischen Fragen gehörend - sich sprachlicher Erfassung entzieht: „Daß etwas an ihr [der Welt] problematisch ist, was wir ihren Sinn nennen. Daß dieser Sinn nicht in ihr liegt, sondern außer ihr (TB, 11.6.16. Vgl. TLP, 6.41). Unmittelbar danach äußert Wittgenstein seine totale Abhängigkeit von der Welt und ermahnt sich, ohne den Versuch eines Einflusses auf die Geschehnisse sich dem Schicksal zu ergeben, um sich so unabhängig von ihm zu machen, also Freiheit im spinozistischen Sinne der Erkenntnis der Notwendigkeit zu erlangen. Denn durch die Abhängigkeit von der äußeren Welt fehlt ihm die innere Ruhe und Gelassenheit für seine Arbeit, über die er im verschlüsselten Teil regelmäßig Protokoll führt. Mit Eintragungen wie „Etwas gearbeitet“, „wenig gearbeitet" usw. dokumentiert er Fortschritte oder Phasen der Unfähigkeit, zu arbeiten. Da er manchmal ,viel gearbeitet" notiert, obwohl im philosophischen Teil nichts geschrieben steht, kann man annehmen, dass er unter „Arbeiten“ auch bloße „Denkarbeit" verstand. In diesem Zusammenhang ist es erwähnenswert, dass Wittgenstein beispielsweise am 8.7.1916 seine Reflexionen über Gott und den Sinn des Lebens, über Zeit und Gegenwart, über das Gewissen sowie über die Übereinstimmung mit der Welt in Normalschrift einträgt, und am selben Tag im verschlüsselten Teil bemerkt „Leider, leider! Ich habe keine Ruhe zum arbeiten!“ Es stellt sich daher die Frage, ob er diese sozusagen metaphysischen Gedankengänge als nicht zur philosophischen Arbeit gehörig betrachtete - was er ja auch im Tractatus und an anderen Stellen seiner Schriften betonte. Dem spräche wieder die Tatsache entgegen, dass er diese Gedanken über metaphysische Probleme 
später in den Tractatus, also in seine philosophische Arbeit, aufnimmt. Überhaupt ist dort eine Vielzahl von Gedanken aufgenommen, die die letzten Seiten der in Normalschrift gehaltenen Tagebücher bestimmten, ihre Anfänge oder Wurzeln, so würde ich sagen, jedoch - und viel früher - in den verschlüsselten Eintragungen nahmen. Doch auch andere Aspekte wie die der Abhängigkeit von der Welt und des Lebens in der Gegenwart, über die Wittgenstein zuerst im persönlichen Teil reflektiert und die dann zum Gegenstand seiner philosophischen Gedankengänge werden, kommen im Tractatus vor.

Meines Erachtens nahm er diese, in der Rezeption oft als mystisch bezeichneten Bemerkungen, in seine philosophische Arbeit auf, um dort den Unterschied zu den rein philosophischen Aufzeichnungen - aber auch ihre Bedeutung innerhalb dieser - zu betonen. Hier allerdings nicht mehr in verschlüsselter Schrift, sondern als Teil des Ganzen, sich lediglich durch die inhaltliche Aussage abhebend.

Seine mühsame Denkarbeit beschreibt Wittgenstein häufig mit Metaphern und unter Einbeziehung des ihn umgebenden Kriegsschauplatzes: so betrachtet er ein gerade ihn beschäftigendes philosophisches Problem als „Belagerung“, oder als „Festung“, die er zu stürmen gedenkt. Mehrmals kommt der Ausdruck ,erlösendes Wort“ vor - zuerst im verschlüsselten Teil, später auch im philosophischen. Am 21.11.1914 notiert er in Code: „Ziemlich gearbeitet. Aber noch immer kann ich das eine erlösende Wort nicht aussprechen. Ich gehe rund um es herum und ganz nahe aber noch konnte ich es nicht selber erfassen!!“

Am darauffolgenden Tag schreibt er im philosophischen Teil, dass er wieder einmal etwas auszudrücken versuche, ,was sich nicht ausdrücken läßt“. Am selben Tag heißt es im verschlüsselten Teil: „Das erlösende Wort nicht ausgesprochen. Gestern lag es mir einmal ganz auf der Zunge. Dann aber gleitet es wieder zurück. - - “ “

Im philosophischen Teil der Tagebücher kommt der Begriff des erlösenden Wortes erst am 20.1.15 vor und steht als Frage nach einer Reihe logischer Gedankengänge: „Das erlösende Wort - ?!“ Am 3.6.15, als er sich intensiv mit der Tautologie von Sätzen auseinandersetzt, schreibt er die Bemerkung „Das erlösende Wort ist übrigens hier noch nicht gesprochen" in Klammern. 
Dieser Ausdruck sollte noch in späteren Jahren in seinen Schriften vorkommen und ist in Zusammenhang mit seiner philosophischen Arbeit zu sehen - als Ziel in seiner Suche nach Lösung philosophischer Probleme - dabei aber auch als eine Art „Erlösung“ in dem verzweifelten Bemühen um den richtigen Ausdruck, das treffende, ,erlösende“ Wort. ${ }^{7}$ Doch ist die religiöse Bedeutung hinsichtlich seiner persönlichen Lage nicht auszuschließen, vielmehr anzunehmen, wenn man diesen Begriff im Kontext der verschlüsselten Tagebuchaufzeichnungen verfolgt. Dies gilt für eine Reihe weiterer Aspekte, die ihren Ausgangspunkt im persönlichen Erleben nahmen und demnach zuerst im verschlüsselten Teil erörtert wurden.

1916, als er an der Front unter steter Lebensgefahr zu kämpfen hatte, sein Wunsch zu leben aber wächst - wofür er sich den Vorwurf einer, falschen Lebensauffassung" macht -, kreisen seine Gedanken im philosophischen Teil um ethische Fragen des Wollens und Nichtwollens, um die Welt des Glücklichen im Gegensatz zur Welt des Unglücklichen. Persönliche Gedankengänge werden deutlich in philosophische übertragen: „Denn daß der Wunsch mit seiner Erfüllung in keinem logischen Zusammenhang steht, ist eine logische Tatsache. Und daß die Welt des Glücklichen eine andere ist als die des Unglücklichen, ist auch klar.“(29.7.17. Vgl. auch TLP, 6.43). Und in seiner Auseinandersetzung mit der Frage des Wollens und Nichtwollens, des Wünschens und Nichtwünschens, meint er - ganz wie im verschlüsselten Teil angedeutet - dass das Nichtwünschen in einem gewissen Sinne das einzig Gute sei.

Die schließlich im Tractatus sozusagen zur „Essenz“ komprimierten Sätze sollten daher aus dem Geist der ihnen vorausgegangenen Tagebücher gelesen werden. ${ }^{8}$ Diese wiederum enthalten wesentliche Aspekte, die meines Erachtens aus den codierten Eintragungen dieser Zeit entwickelt worden sind. Sie geben Wittgensteins ganz persönliche Sicht auf verschiedene Themen wieder, vor allem hinsichtlich existentieller Fragen sowohl des Individuums als auch des Menschen an sich in seinem sozialen

${ }^{7}$ Vgl.: ,Die Aufgabe der Philosophie ist es, das erlösende Wort zu finden. Das erlösende Wort ist die Lösung eines philosophischen Problems“" (MS 107, 114). Vgl. auch: „Der Philosoph trachtet das erlösende Wort zu finden, das ist das Wort das uns endlich erlaubt das zu fassen was bis jetzt immer ungreifbar unser Bewußtsein belastet hat" (MS 110, 17).

${ }^{8}$ Vgl. auch Schulte 2001, 211. 
und kulturellen Umfeld. Im Hinblick auf die Bedeutung der Einzigartigkeit des individuellen Bewusstseins und Wahrnehmens der Welt möchte ich eine Eintragung vom 1.8.1916 zitieren: „Nur aus dem Bewußtsein der Einzigkeit meines Lebens entspringt Religion - Wissenschaft - und Kunst.“

\subsection{Tagebücher 1930-1932/1936-1937 (Denkbewegungen)}

Die codierten Bemerkungen dieser Tagebücher betreffen vor allem Äußerungen über moralische und religiöse Probleme, während allgemeine persönliche Eintragungen in Normalschrift verfasst sind. Auch Reflexionen kulturgeschichtlicher Art, die im übrigen Nachlass häufig in Code geschrieben sind, erscheinen hier in Normalschrift. Zudem gibt es einige philosophische Aufzeichnungen über die Idee bzw. das „Urbild“ oder „Zeichen" usw. - Sätze, die in sehr ähnlicher Form in den Philosophischen Untersuchungen vorkommen. Allerdings sind dies Fragen, die den metaphysischen Bereich berühren, den Charakter des Sich-zeigenden tragen, und Wittgenstein verwendet dabei auch Wörter wie „unsagbar“, „Geste“ und dergleichen. So gesehen gilt für dieses Tagebuch die These, dass Wittgenstein metaphysische, die phänomenale Welt transzendierende Fragen auch innerhalb seiner Tagebücher behandelte, für seine ganz persönliche Auseinandersetzung mit Ethik und Religion zudem den Code verwendete - Fragen, die er für wesentlich befand, vielleicht auch für zu „kostbar“ oder „sublim“, um für den flüchtigen, oberflächlichen Leser ohne Anstrengung verfügbar anstatt verborgen zu sein. Dafür spräche ja auch die anfangs zitierte Metapher von der Schatulle. Auffallend ist, dass diese Bemerkungen in stilistischer und inhaltlicher Hinsicht den verschlüsselten Tagebüchern der Kriegsjahre gleichen, manchmal dem Wortlaut identische Sätze enthalten. Es handelt sich dabei um leidenschaftliche Gebete und Anrufungen an Gott, von der Art wie: „Möge Gott mir helfen.“, „Erhalte mir meinen Verstand rein \& unbefleckt!“ (DB, 174), oder „Hilf \& Erleuchte!“ (DB, 223). Die Ängste, den Verstand zu verlieren und dem Wahnsinn zu verfallen, sind ebenfalls in Code geschrieben.

Ein Beispiel sei angeführt, wo Wittgenstein bei der Schwierigkeit rationaler Erklärungen an die Möglichkeit des Beschreibens denkt, dabei von normaler zu verschlüsselter Schrift wechselt, sobald es um das persönliche Empfinden seiner geistigen Überbeanspruchung geht: „Nicht erklä$\underline{\text { ren! }}$ - Beschreiben! Unterwirf dein Herz \& sei nicht bös, dass du so leiden 
musst! Das ist der Rat, den ich mir geben soll. Wenn du krank bist, dann richte dich in dieser Krankheit ein; sei nicht bös dass du krank bist "“ (DB, 183f.).

Die in Code geführten Eintragungen betreffen Gedanken und Empfindungen, die Wittgenstein besonders nahe zu gehen scheinen, während der Großteil dieses Tagebuchs in Normalschrift gehalten ist - wahrscheinlich, da es von vornherein als solches konzipiert war und er daher den Code nicht für nötig erachtete, den er sonst für persönliche oder allgemein kulturwissenschaftliche Aufzeichnungen innerhalb philosophischer Texte anwandte, um diese davon abzugrenzen.

Auf den Unterschied zwischen persönlichen Äußerungen religiöser Art und jenen, für Andere bestimmte, also allgemeine, an Theorien grenzende, hat er selbst hingewiesen: „Es ist ein Ding zu Gott zu reden \& ein anderes, von Gott zu Anderen zu reden“(DB, 174).

Seine verschlüsselten, in leidenschaftlichem Ton gehaltenen Aufzeichnungen im Gegensatz zu den nüchtern geführten Disputen in den philosophischen Manuskripten oder etwa in den Vorlesungen über Religion ${ }^{9}$ belegen diese Unterscheidung.

\section{Codierte Bemerkungen ethischen und religiösen Inhalts}

Hinsichtlich seiner Zurückhaltung gegenüber ethischen und religiösen Fragen als Gegenstand philosophischer Erörterung scheint die häufige Befassung mit dieser Thematik in verschlüsselter Schrift ein Indiz dafür zu sein, dass er diese aus der Philosophie heraushalten und auf einer anderen Ebene - der Ebene des Zeigens, nicht des Erklärens - betrachten wollte. Ein interessantes Beispiel in diesem Zusammenhang ist die Tatsache, dass er die Bemerkung ,Wenn etwas Gut ist so ist es auch Göttlich. Damit ist seltsamerweise meine Ethik zusammengefasst" (MS 107, 192; 10.11.1929) in verschlüsselter Schrift, die nur vier Tage später geschriebene Bemerkung „Man kann die Menschen nicht zum Guten führen; man kann sie nur irgendwohin führen; das Gute liegt außerhalb des Tatsachenraumes“" (MS 107, 196; 15.11.1929) in Normalschrift eintrug. Denn der obigen Bemerkung liegt eine Wertung bezüglich seiner Auffassung von Ethik inne, die

\footnotetext{
${ }^{9}$ Vgl. Lectures and Conversations on Aesthetics, Psychology and Religious Belief.
} 
einer Theorie gleichkommt. Daher wohl das Verbergen mittels Verschlüsselung. Die spätere Bemerkung hingegen stellt keine Theorie dar, sondern lediglich die resignierende Einsicht der Unmöglichkeit ethischer Richtlinien innerhalb der Welt der Tatsachen. Und wie aus dem Tractatus bekannt, betont er, dass Werte wie das Gute außerhalb des Tatsachenraumes, also außerhalb des rational Erfassbaren, liegen.

Die häufige Eintragung ethischer und religiöser Reflexionen in verschlüsselter Schrift lässt vermuten, dass Wittgenstein für diesen Bereich, wie erwähnt, einen eigenen Texttypus kreierte, der sich vom philosophischen abheben, dabei aber gerade durch die Technik des Verhüllens auf den Aspekt des Verborgenen, nicht Sichtbaren hinweisen sollte. So dass die seiner Ansicht nach wesentlichen Fragen zwar berührt, aber nicht zum Gegenstand eines philosophischen Disputes gemacht würden, und durch ihre Codierung in einen separaten Teil seines Werkes verwiesen werden, über den er im offiziellen philosophischen Teil geschwiegen hat. Sätze ethischen und religiösen Inhalts in Code könnten somit als symbolisch für das Nicht-Sagbare, nur Zeigbare gesehen werden. Zugleich wird der Unterschied zwischen „sinnvollen“ und „unsinnigen“ Sätzen hervorgehoben, anhand von unterschiedlichen Schriftarten betont. Dies wird besonders in den philosophischen Manuskripten deutlich, wo codierte Bemerkungen ethischen, religiösen oder auch ästhetischen Inhalts inmitten nüchterner philosophischer Überlegungen als etwas Eigenständiges herausragen. Die Annahme, diese aus der Philosophie heraushalten zu wollen, wird dadurch erhärtet, dass er die codierten Eintragungen vor der 1929 begonnenen Übertragung der Notizbücher in Bände in eckigen Klammern gesetzt hatte (vgl. Pichler 1997, 68f.).

\section{3. Über den Nachlass verstreute Bemerkungen}

Obwohl eine Vielzahl dieser Aufzeichnungen in den Vermischten Bemerkungen publiziert sind, so geschah dies anderen Kriterien gemäß, d.h. vorwiegend nach inhaltlichen, und dies im speziellen nach Themen kulturgeschichtlicher Art. Zudem befinden sich in den Vermischten Bemerkungen auch zahlreiche, in Normalschrift verfasste Stellen, während eine beträchtliche Menge weiterer verschlüsselter Stellen aus dem Nachlass fehlt. 
Die Frage stellt sich, welche Bedeutung diesen codierten Stellen innerhalb der philosophischen Manuskripte zukommt, und ob sich Zusammenhänge zwischen diesen Äußerungen und dem philosophischen Werk feststellen lassen? Ob diese Bemerkungen vielleicht als Beispiel für Wittgensteins Sprachphilosophie angesehen werden können - sofern er mit ihnen eine bestimmte Funktion von Sprache erfüllt sah, die durch wissenschaftliche Dispute nicht erreicht wird? Hat er diese aphorismenhaften, teils lyrisch anmutenden Bemerkungen inmitten des fortlaufenden nüchternen philosophischen Diskurses gesetzt, um den Unterschied zwischen verschiedenen Sprachmöglichkeiten - man könnte sogar sagen, Sprachspielen - aufzuzeigen? Als eine besondere Art, Sich-Zeigendes von klar Sagbarem zu trennen? Etwa in Form von einer Art poetischer Sätze als Gegenpol zu streng philosophischen Argumentationen? Und lässt sich, wie erwähnt, eine Parallele zu Wittgensteins wiederholten Hinweisen auf die Bedeutung von Mimik, Gesten und Gebärden ziehen, die sprachliche Erklärungen in seinen philosophischen Untersuchungen ersetzen sollten? Konkret: Was bedeuten codiert verfasste Äußerungen wie die folgende in Zusammenhang mit Wittgensteins philosophischem Denken?

O, warum ist mir zumute, als schrieb ich ein Gedicht, wenn ich Philosophie schreibe? Es ist hier, wie wenn hier ein Kleines wäre, das eine herrliche Bedeutung hat. Wie ein Blatt, oder eine Blume (MS 133, 31.10.1946).

Diese Stelle findet sich inmitten seiner Auseinandersetzung mit der Bedeutung von Namen innerhalb von Sprachspielen. Nach dem angeführten Zitat schreibt er: „Die Sucht nach Erklärung ist der vollen Auffassung der Tatsachen, also der Beschreibung, hinderlich. Die vorgefaßte Hypothese wirkt wie ein Sieb, das nur einen ganz kleinen Teil der Tatsachen zu unserer Betrachtung gelangen läßt.“"

An anderer Stelle, im MS 146, allerdings in Normalschrift und demnach in vergleichbar nüchternem, vom Lyrischen sich abhebenden Ton - als eine Art Fazit über seine philosophische Arbeit und daher Information für all jene, die sich mit seiner Philosophie befassen - heißt es: "Ich glaube meine Stellung zur Philosophie dadurch zusammengefaßt zu haben indem ich sagte: Philosophie dürfte man eigentlich nur dichten." 
Diese und weitere Bemerkungen über sein Philosophieren lassen vermuten, dass er inmitten angestrengter Gedankengänge über philosophische Probleme zeitweise innehielt, um sozusagen Atem zu holen, und dann, bei der Schwierigkeit, diese Probleme auf analytische Weise zu lösen, sich anderer Formen des Umgangs mit Sprache und Philosophie bewusst wurde - Formen, die eher zeigender als erklärender bzw. sagender Art sind. Da diese Bemerkungen jedoch den eigentlichen philosophischen Diskurs unterbrechen, zu diesem gar nicht gehören, so grenzt er sie durch eine eigene Schriftart von dem in Normalschrift gehaltenen Prozess seiner philosophischen Untersuchungen ab. Doch nicht nur die unterschiedliche Schriftart der Code - , sondern auch der Ton, der Stil erweist sich als ein anderer als der nüchtern argumentierende Ton seiner philosophischen Arbeit. Diese eingeschobenen Reflexionen über seine Art des Philosophierens wirken wie Kommentare aus einer Meta-Ebene, hinterfragen die Richtigkeit oder Notwendigkeit seiner philosophischen Argumentationen.

Während die mehr oder weniger strikte Trennung zwischen philosophischen und persönlichen/ethischen/religiösen Eintragungen gemäß normaler und verschlüsselter Schrift insbesondere für die Tagebücher 1914-16 gilt, lässt sich hinsichtlich der in den philosophischen Manuskripten verstreuten codierten Bemerkungen eine Art Ineinanderüberfließen der zwei Textsorten, ein Verwischen dieser Grenzen beobachten: d.h. es finden sich Bemerkungen ethischen und religiösen Inhalts manchmal in Code, manchmal in Normalschrift. Dies betrifft vor allem die Jahre 1931 und 1936/37 - die Zeit, als Wittgenstein sich verstärkt dem Tagebuchschreiben widmete und sogar den Gedanken einer Autobiographie erwog. Interessanterweise trug er seine Reflexionen über das Judentum in Normalschrift ein. Meiner Vermutung nach wollte er im Hinblick auf autobiographisches Schreiben sowie das Ziel einer Beichte ${ }^{10}$ diese Angelegenheiten nicht länger mittels Code verbergen, sondern, gemäß seinem Ideal einer wahrheitsgemäßen Autobiographie, das Insgesamt seiner Persönlichkeit einschließlich negativer Seiten seines Charakters für jeden einsichtig machen. Nur sehr persönliche, direkt an Gott gerichtete Eintragungen wie die in leidenschaftlichem

${ }^{10}$ Laut Drury war dies 1931, schriftlichen Zeugnissen zufolge jedoch erst Ende 1936 (in Briefen an Ludwig Hänsel) und Anfang 1937 (gegenüber Freunden in England). 
und zugleich demütigem Ton gehaltenen Gebete, zumeist mit der aus den Kriegstagebüchern bekannten „Möge-Form“, schrieb er in Code: „Möge Gott es verhüten!“ (MS 108, 38; 25.12.1929); „Gott, halte mein Ideal zurecht!“" (MS 107, 161; 11.10.1929). Oder, später: „Möchte mir Gott Reinheit \& Wahrheit schicken" (MS 108, 47; 28.12.29, 47).

Es scheint, dass hier der Code als Mittel diente, um sein von ehrfürchtiger Distanz geprägtes Verhältnis zu Gott auszudrücken, und dabei das in normaler Sprache nicht Artikulierbare - die Unsinnigkeit ethischer und religiöser Ausdrücke - zu verbergen. Und wenn auch zahlreiche Reflexionen über religiösen Glauben in Normalschrift festgehalten sind, geht Wittgenstein zum Code über, sobald sein Ton persönlich und leidenschaftlich wird:

Wenn ich aber wirklich erlöst werden soll, - so brauche ich Gewissheit - nicht Weisheit, Träume, Spekulation - und diese Gewissheit ist der Glaube. Und der Glaube ist Glaube an das, was mein Herz, meine Seele braucht, nicht mein spekulierender Verstand, Denn meine Seele, mit ihren Leidenschaften, gleichsam mit ihrem Fleisch \& Blut muss erlöst werden, nicht mein abstrakter Geist (MS 120; 12.12.1937).

Während seines langen einsamen Aufenthalts in Norwegen in den Jahren 1936 und 1937 schreibt er längere Passagen in Code, insbesondere was seine persönliche Lage betrifft, womit sich einer der von ihm angeführten Gründe für das Tagebuchschreiben bestätigt - nämlich als Ersatz für einen Menschen, dem er sich anvertrauen könnte. Er berichtet über seine Ängste, dem Wahnsinn zu verfallen, seinen Zustand äußerster Verzweiflung. Häufig bezichtigt er sich der Unanständigkeit, Eitelkeit, des Mangels an Wahrheitsliebe und der Feigheit. Wenn sich seine Gedankengänge von seiner persönlichen Lage zu allgemeinen Problemen des Lebens erstrecken, ändert sich sein Ton, d.h. von einem leidenschaftlichen zu einem eher nüchternen, wie er uns aus den philosophischen Tagebüchern 1914-16 bekannt ist. Der monologische Charakter weicht einem dialogischen:

Die Lösung des Problems, das Du im Leben siehst, ist eine Art zu leben, die das Problemhafte zum Verschwinden bringt. Dass das Leben problematisch ist, heisst, dass Dein Leben nicht in die Form des Lebens passt. Du musst dann dein Leben verändern, \& passt es in die Form, dann verschwindet das Problematische $[\ldots]$ “. (MS 118, 17r. 27.8.1937). 
Oft berichtet er in Code über seine philosophische Arbeit, so dass wir ein lebhaftes Bild der Beweggründe und der Art seiner zahlreichen Änderungen, Streichungen etc. erhalten.

Er beklagt seine Unfähigkeit, klar zu denken und seine philosophischen Untersuchungen übersichtlich darzustellen, und befürchtet einen allmählichen, aber völligen Verlust seines Verstandes, ein Schwinden seiner Geisteskraft (1.10.1940). In der Schilderung des Zustands vom Nachlassen philosophischer Denkkraft verwendet er Metaphern wie „Öde“, „Winter", „Wüste“ und dergleichen. Das Hauptproblem seiner Verzweiflung sieht er jedoch in seiner Vereinsamung. Häufig macht er sich nun Gedanken über die Liebe, die er als das Wichtigste im Leben sieht, als einen „seltenen Edelstein“ (MS 132, 77; 29.9.1946), als „die Perle von grossem Wert“ (MS 133, 8r; 26.10.1946), als ,ein Glück. Vielleicht ein Glück mit Schmerzen, aber ein Glück“ (ibid.).

\section{Konklusion}

Wie sich aus meinen Ausführungen gezeigt hat, trifft die These von Sagbarem und Zeigbarem hinsichtlich Normalschrift und Code in Wittgensteins Nachlass auf die meisten, allerdings nicht auf alle Stellen zu. Dass er jedoch mittels Verschlüsselung einen eigenen Texttypus in seinem Oeuvre anstrebte, um den Unterschied zu strikt philosophischen Aufzeichnungen hervorzuheben, scheint trotz nicht durchgängig feststellbarer Einhaltung sei es aufgrund von Nachlässigkeit in der Eile des Schreibens oder anderer Faktoren - offensichtlich. Dafür sprechen nicht nur inhaltliche und stilistische Unterschiede, sondern auch die Tatsache, dass er, wie erwähnt, die in Code geschriebenen Bemerkungen zuerst in Normalschrift, aber in eckige Klammern setzte. Als hätte er den seiner Auffassung nach in der Philosophie nicht zu behandelnden Fragen einen eigenen, nicht unmittelbar zugänglichen Raum zuweisen wollen, und dafür die Form der Verschlüsselung gewählt.

Trotz offenbarer Inkonsequenz scheint der Code hinsichtlich seiner Bemerkung „Die Grenzen meiner Sprache bedeuten die Grenzen meiner Welt" (TLP, 5.6) ihm als Medium gedient zu haben, um über die Grenzen seiner Sprache im Sinne der artikulierbaren, verbalisierbaren Welt hinaus- 
zugehen - ein Wagnis, bei dem ihm allerdings die Unsinnigkeit des zur Sprache Gebrachten bewusst geworden ist.

Im Hinblick auf seine Bemerkung über die innerhalb der Grenzen seiner Sprache geäußerten Sätze im Tractatus - dass man diese, wenn man ihn verstehe, am Ende als unsinnig erkenne (TLP, 6.54) -, könnten die verschlüsselten, ethische und religiöse Fragen betreffenden Bemerkungen aber auch als zum wesentlichen, nicht geschriebenen Teil seines Werks ${ }^{11}$ gehörend gedacht sein.

\section{Literatur}

Baum, Wilhelm (Hg.) 1990: Ludwig Wittgenstein. Geheime Tagebücher 1914-1916. Wien: Turia \& Kant.

Drury, Maurice O’Connor 1992: „Bemerkungen zu einigen Gesprächen mit Wittgenstein“. In: Rhees 1992, 117-141.

McGuinness, Brian 1992: Wittgensteins frühe Jahre. Übersetzt. von Joachim Schulte. Frankfurt: Suhrkamp.

Pichler, Alois 1993: „Wittgensteins spätere Manuskripte: einige Bemerkungen zu Stil und Schreiben." In: Mitteilungen aus dem Brenner-Archiv 12, 8-26.

Pichler, Alois 1997: „Wittgenstein und das Schreiben: Ansätze zu einem Schreiberporträt.“ Diplomarbeit. Innsbruck.

Pichler, Alois / von Wright, G. H. / Nyman, Heikki (Hg.) 1994: Ludwig Wittgenstein. Vermischte Bemerkungen. Frankfurt: Suhrkamp.

Rhees, Rush (Hg.) 1992: Ludwig Wittgenstein: Porträts und Gespräche. Frankfurt: Suhrkamp.

Schulte, Joachim 2001: „Ich bin meine Welt“. In: Arnswald, Ulrich / Weiberg, Anja (Hg.): Der Denker als Seiltänzer. Wittgenstein über Religion, Mystik und Ethik. Düsseldorf: Parerga, 193-213.

Schulte, Joachim (Hg.) 1989: Ludwig Wittgenstein. Vortrag über Ethik und andere kleine Schriften. Frankfurt: Suhrkamp.

${ }^{11}$ Vgl. dazu Wittgensteins Brief an Ludwig von Ficker [Anf. Nov. 1919], in dem er schrieb, dass sein Werk aus zwei Teilen bestehe: ,aus dem, der hier vorliegt, und aus alledem, was ich nicht geschrieben habe. Und gerade dieser zweite Teil ist der Wichtige“. 
Schopenhauer, Arthur 1977: Werke in zehn Bänden. Zürcher Ausgabe. Hg. von Angelika Hübscher. Diogenes.

Spinoza, Baruch de 1955: Die Ethik. Schriften. Briefe. Hg. von Friedrich Bülow. Stuttgart: Kröner Verlag.

Somavilla, Ilse (Hg.) 1997: Ludwig Wittgenstein. Denkbewegungen. Tagebücher 19301932/1936-1937. Innsbruck: Haymon. (DB)

Somavilla, Ilse (Hg.) 2006: Wittgenstein - Engelmann. Briefe, Begegnungen, Erinnerungen. Hg. von Ilse Somavilla unter Mitarbeit von Brian McGuinness. Innsbruck: Haymon. (Engelmann)

Tolstoi, Leo N. 1892: Kurze Darlegung des Evangelium. Aus dem Russischen von Paul Lauterbach. Leipzig: Reclam.

Wittgenstein, Ludwig 1966: Lectures and Conversations on Aesthetics, Psychology and Religious Belief. Ed. by Cyril Barrett. Oxford: Blackwell.

Wittgenstein, Ludwig 1969: Briefe an Ludwig von Ficker. Hg. von G.H. von Wright unter Mitarbeit von Walter Methlagl. Salzburg: Otto Müller Verlag.

Wittgenstein, Ludwig 1984: Philosophische Untersuchungen. In: Werkausgabe, Bd. 1. Frankfurt: Suhrkamp.

Wittgenstein, Ludwig 1984:. Tagebücher 1914-1916. In: Werkausgabe, Bd. 1. Frankfurt: Suhrkamp.

Wittgenstein, Ludwig 1984: Tractatus logico-philosophicus. In: Werkausgabe, Bd. 1. Frankfurt: Suhrkamp.

Wittgenstein, Ludwig ${ }^{3}$ 1989: Ludwig Wittgenstein und der Wiener Kreis. Gespräche aufgezeichnet von Friedrich Waismann. In: Werkausgabe, Bd. 3. Frankfurt: Suhrkamp. Wittgenstein, Ludwig 2000: Wittgenstein's Nachlass. The Bergen Electronic Edition. Bergen / Oxford: Oxford University Press. 